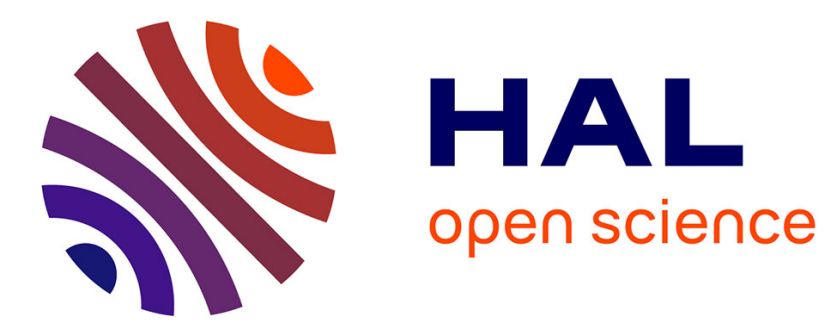

\title{
Engagement et persuasion par la peur: vers une communication engageante dans le domaine de la santé
}

\author{
Fabien Girandola, F. Michelik
}

\section{To cite this version:}

Fabien Girandola, F. Michelik. Engagement et persuasion par la peur: vers une communication engageante dans le domaine de la santé. Revue canadienne des sciences du comportement, 2008, 40, pp.69-79. 10.1037/0008-400X.40.2.69 . hal-00568210

\section{HAL Id: hal-00568210 \\ https://u-bourgogne.hal.science/hal-00568210}

Submitted on 13 Apr 2011

HAL is a multi-disciplinary open access archive for the deposit and dissemination of scientific research documents, whether they are published or not. The documents may come from teaching and research institutions in France or abroad, or from public or private research centers.
L'archive ouverte pluridisciplinaire HAL, est destinée au dépôt et à la diffusion de documents scientifiques de niveau recherche, publiés ou non, émanant des établissements d'enseignement et de recherche français ou étrangers, des laboratoires publics ou privés. 


\title{
Engagement et persuasion par la peur : vers une communication engageante
}

\section{Dans le domaine de la sante}

\author{
Fabien Girandola \& Fabienne Michelik
}

\begin{abstract}
Depuis plus de cinquante ans, des recherches ont montré les effets de la peur sur les attitudes et les comportements. L'expérience rapportée dans cet article visait à tester l'effet de l'engagement sur une tentative de persuasion ultérieure, en l'occurrence après la lecture d'un message anti-alcool. II s'agissait d'amener des buveurs d'alcool, faiblement et fortement engages dans leur comportement de consommation, à modifier leur attitude et leur intention comportementale envers l'alcool. Conformément aux attentes, les buveurs fortement engages (c.-à-d., des buveurs habituels) ont résisté à la persuasion tant sur le plan de l'attitude que de l'intention. Les participants faiblement engages (c.-à-d., des buveurs occasionnels) n'ont pas résisté' a' la persuasion et ont adopté une attitude plus favorable envers l'alcool. Les buveurs ayant lu un message fortement menaçant ainsi que fortement rassurant ont exprimé la plus forte intention de réduire leur consommation d'alcool. Une discussion des résultats est présentée à la lumière du modèle des réponses parallèles étendu de Witte (1998). Une nouvelle perspective est présentée à la fin de l'article : la communication engageante.
\end{abstract}

Mots clés : engagement, persuasion, communication engageante, peur, santé

Les recherches sur la santé publique font valoir l'intérêt de construire des messages persuasifs et efficaces. La recherche tente de promouvoir des messages susceptibles de susciter des changements attitudinaux et comportementaux (Eagly \& Chaiken, 1993; Girandola, 2003). Toutefois, les résultats obtenus, pour ce qui a trait au changement des attitudes ou des comportements, sont souvent loin de satisfaire les concepteurs de campagnes de prévention. La persuasion et l'engagement constituent les deux principaux champs d'étude du changement. La littérature sur la persuasion offre une palette de variables et de paramètres susceptibles d'influencer les attitudes et les comportements des individus (Crano \& Prislin, 2006; Girandola, 2000b; 2002; Johnson, Maio \& Smith-McLallen, 2005; Perloff, 2001, 2003; Petty, Priester \& Brinõl, 2002). Par exemple, les résultats de dizaines de recherches expérimentales ou appliquées montrent que la peur peut agir efficacement sur les attitudes et les comportements (par ex., Girandola \& Atkinson, 2003; Witte \& Allen, 2000). La littérature sur l'engagement nous informe, quant à elle, sur les techniques d'influence susceptibles de déboucher sur un changement social (Girandola, 2005; Girandola \& Roussiau, 2003; Joule \& Beauvois, 1998, 2002; Olson \& Stone, 2005). Elle fournit aussi des éléments permettant de mieux comprendre l'effet de messages persuasifs : 
Un individu ayant un comportement dans un contexte fortement engageant (libre choix, aucune sanction, faible récompense) résistera mieux à une tentative de persuasion ultérieure (Kiesler, 1971). Ces deux champs de recherche permettraient, selon nous, une meilleure compréhension du changement et une prédiction plus efficace de la résistance au changement. L'objectif de cet article est de proposer une articulation expérimentale et appliquée de ces deux champs, traditionnellement disjoints. Notre recherche a pour objectif la construction d'un message à intention persuasive utilisant la peur ainsi que la mesure de ses effets sur des individus buveurs habituels (fort engagement à l'égard de la consommation d'alcool), par opposition à des buveurs occasionnels (faible engagement) à l'égard de la consommation d'alcool). Les critères de mesure seront le changement d'attitude envers l'alcool et l'intention de réduire sa consommation d'alcool. A la fin de l'article, nous présenterons des pistes de recherche ainsi qu'un nouveau paradigme : la communication engageante.

\section{Alcool : statistiques, conséquences sociales et effets sur la santé}

En France, I'alcool représente un problème de santé publique (par ex., Cerclé, 2002). Quelques données (Observatoire français des drogues et des toxicomanies) sont révélatrices : $96 \%$ des jeunes de 18 à 25 ans ont expérimenté l'alcool, et $15 \%$ d'entre eux disent consommer régulièrement de l'alcool (au moins trois fois par semaine). Parmi les adultes, 14 \% déclarent avoir été en état d'ébriété au moins une fois au cours des 12 derniers mois. L'alcool est un facteur relié à un grand nombre de pathologies : cancers des voies aérodigestives et du foie, et maladies de l'appareil circulatoire. Il est souvent en cause dans les accidents et les suicides. En France, en 1995, on estimait à 45000 le nombre de décès attribuables à l'alcool (OFDT, 2002). L'alcool favorise aussi les relations sexuelles sans protection (par ex., MacDonald, Zanna \& Fong, 1998), suscite le plus souvent un sentiment d'invulnérabilité (par ex., Goldberg, Halpern-Felsher \& Millstein, 2002) et accentue la prise de risques (Boscher, Pavic \& Pérrissol, 2002). Le comportement et l'image du buveur sont valorisés (Chassin, Tetzloff \& Hershey, 1985) et peuvent faire l'objet de mimétisme et d'influence entre jeunes (Andrews, Tildesley, Hops \& Li, 2002). L'alcool est souvent à l'origine d'actes d'agression directs ou indirects (Cerclé, 2000; Giancola \& Zeichner, 1997), d'une délinquance (par ex., conduites en état d'ébriété, homicides, crimes et délits sexuels, outrages, dégradation d'objets d'utilité publique). Les appels àla peur et leurs applications.

A la suite des travaux de Leventhal (1970) et de Rogers $(1983)$, Witte $(1992,1994)$ a proposé le «Modèle étendu des processus parallèles ». Selon ce dernier (1998), un message utilisant la peur comporte deux parties. La première informe d'une menace potentielle (possibilité d'un cancer) en insistant sur la sévérité de cette menace et la vulnérabilité de la population concernée. La seconde propose des recommandations efficaces pour faire face à cette menace. Elle préconise soit un comportement de détection (subir une radiographie des poumons), soit de prévention (diminuer ou arrêter sa consommation de tabac). Le message souligne ensuite la facilité de la mise en oeuvre de ces recommandations et insiste sur la 
capacité des individus à le faire (auto-efficacité ou "selfefficacy "). L'auto-efficacité augmente la perception de maîtrise, c'est-à-dire la confiance dans les stratégies de protection disponibles et dans la facilité de leur mise en oeuvre. Le modèle de Witte (1998) reprend la distinction soulignée par Leventhal (1970) entre le contrôle du danger et la gestion de la peur. Witte a pu montrer que les individus se livrent à une évaluation du rapport entre l'efficacité (efficacité des recommandations et autoefficacité ) et la menace (sévérité et vulnérabilité). Si ce rapport est en faveur de l'efficacité, les individus adoptent des comportements de prévention adaptés à la menace (contrôle du danger). A l'inverse, si ce rapport met en relief la supériorité de la menace (les recommandations proposées paraissent inefficaces ou les sentiments d'auto-efficacité des individus sont faibles), les individus utilisent des stratégies de résistance motivées par un besoin de maîtriser leur peur: déni, rejet du message, adoption de comportements contraires à ceux qui sont recommandés. Witte $(1992,1994)$ a provoqué des changements d'attitudes et de comportements face à la prévention du VIH Les adolescents ont adopté une attitude plus favorable envers l'utilisation des préservatifs et ont manifesté une plus forte intention de les utiliser lorsque la menace, l'efficacité perçue et l'auto-efficacité étaient à leurs plus hauts niveaux. Six semaines plus tard, ces adolescents ont rapporté l'utilisation la plus fréquente de préservatifs. Ces résultats ont été reproduits sur différents thèmes (par ex., prévention des maladies transmises sexuellement, cancer de la peau). Par ailleurs, Moscato, Black, Blue, Mattson, Galer-Unti et Coster (2001) ont montré que la peur est un moyen efficace pour persuader les étudiants à ne plus boire d'alcool. Selon Witte et Allen (2000), les campagnes de prévention devraient mettre davantage l'accent sur la menace tout en renforçant l'efficacité perçue (efficacité des recommandations et auto-efficacité), afin que les individus adoptent les recommandations proposées dans le message.

\section{Comportements antérieurs, engagement et résistance à la persuasion}

Les recherches sur les relations entre l'attitude et le comportement ont conduit à sousestimer l'influence des comportements antérieurs (Jaccard \& Blanton, 2005; Sutton, 1994), des habitudes (Verplanken, Myrbakk \& Rudi, 2005) et des routines (Betsch \& Haberstroh, 2005) sur les comportements ultérieurs. La théorie de l'engagement (Kiesler, 1971; Joule \& Beauvois, 1998) permet de mieux comprendre la relation entre comportement antérieur et comportement ultérieur. L'engagement est " le lien qui unit l'individu à ses actes " (Kiesler, 1971). Cette de'finition a été reformulée par Joule et Beauvois (1998). Ces derniers ont mis en évidence la double caractéristique de cause et d'effet de l'engagement. C'est précisément parce qu'il y a engagement qu'un lien pourra s'établir entre un individu et ses actes. L'engagement est tributaire de la situation : c'est la situation, dans ses caractéristiques objectives, qui engage ou non l'individu dans ses actes et qui favorise, entrave ou interdit l'établissement d'un lien entre l'individu et ses actes. Selon Joule et Beauvois (1998), l'engagement correspond dans une situation donnée « aux conditions dans lesquelles la réalisation d'un acte ne peut être imputable qu'à celui qui l'a réalisé ". Un certain nombre de facteurs assurent l'engagement à l'égard d'un comportement. Ils se 
classent en deux catégories. Dans un premier temps, il y a la visibilité et l'importance de l'acte. Cette catégorie comprend:

a) le caractère public de l'acte - un acte réalisé en public est plus engageant qu'un acte anonyme;

b) le caractère explicite de l'acte - un acte explicite est plus engageant qu'un acte ambigu;

c) l'irrévocabilité de l'acte - un acte irrévocable est plus engageant qu'un acte révocable;

d) la répétition de l'acte - un acte que l'on répète est plus engageant qu'un acte qu'on ne réalise qu'une fois;

e) les conséquences de l'acte - un acte est plus engageant lorsqu'il est lourd de conséquences;

f) le coût de l'acte : un acte est engageant lorsqu'il est coûteux en argent ou en temps.

Dans un deuxième temps, il y a le contexte de liberté et les raisons de l'acte. Cette catégorie comprend:

a) le libre choix, considéré comme la variable fondamentale à l'engagement;

b) les raisons expliquant ce que l'on fait ou ce que l'on s'apprête à faire, lesquelles peuvent être d'ordre externe ou interne. Les raisons externes " désengagent " : elles distendent le lien entre un individu et ses actes. Par exemple, plus les récompenses et les punitions sont fortes, plus elles justifient l'acte.

Quant aux raisons internes, elles resserrent le lien entre un individu et ses actes. II est possible d'augmenter l'engagement en favorisant des auto-attributions internes (par ex., " Vous êtes vraiment quelqu'un de responsable. "). La répétition d'un acte ou sa fréquence est un facteur d'engagement (Joule, 1991a, b; Kiesler, 1971). En matière de consommation d'alcool, une consommation fréquente traduit un comportement engagé. Ainsi, la consommation d'alcool posséderait-elle toutes les caractéristiques d'une conduite engageante, à plus d'un titre. D'abord, elle se répète dans le temps (répétition de l'acte, fréquence de l'acte), elle est librement choisie, elle est parfois publique (au bar, entre amis) et irrévocable (le verre est vide). Par conséquent, les buveurs habituels seraient plus engagés dans leur comportement de consommation d'alcool que les buveurs occasionnels.

Plusieurs résultats d'expériences corroborent cette hypothèse. Ils montrent que les comportements actuels trouvent le plus souvent leur détermination dans des comportements antérieurs répétitifs et engageants pouvant s'assimiler à des habitudes. Sur ce point, la documentation scientifique est imposante. Ouellette et Wood (1998) ont montré que la fréquence de réalisation d'un comportement antérieur est un bon prédicteur du comportement ultérieur. Ronis, Yates et Kirscht (1989), puis Triandis (1980) ont montré qu'une habitude procède de la répétition d'un comportement antérieur ou d'une fréquence 
comportementale élevée. Verplanken et Orbell (2003), puis Verplanken, Aarts et Van Knippenberg (1997) ont pu montrer que la répétition ou la fréquence d'un comportement et l'automaticité qui en résulte peuvent, en partie, participer à la création d'une habitude (voir Verplanken, 2006).

Nous savons qu'un individu engage' dans un comportement n'est guère motivé à traiter l'information persuasive qui est à sa disposition. Cet individu résiste plus facilement à une tentative de persuasion que celui qui est non engagé : l'engagement entrave, voire interdit un traitement approfondi de l'information (Kiesler, 1971). Kiesler et Sakumura (1966) ont été les premiers à tester l'effet de l'engagement sur une tentative de persuasion ultérieure. Ces auteurs ont montré que plus un individu est engagé à l'égard d'un comportement conforme à une de ses attitudes, plus il a tendance à résister à une tentative de persuasion ultérieure remettant en cause cette attitude. Dans ce droit fil, l'expérience de Kiesler et Mathog (dans Kiesler, 1971, p. 66-74) a mis en relief les effets de la répétition d'un acte (facteur d'engagement) sur l'acceptation ultérieure d'une communication persuasive. La répétition ou la fréquence de l'acte traduit, dans ce cas, l'engagement. Selon ces auteurs, la résistance à la persuasion est fonction du nombre d'actes antérieurs en accord ou en relation de consonance avec l'attitude. Plus le nombre d'actes consonants est important, plus forts seront l'engagement et la résistance à une tentative de persuasion ultérieure. Cent cinquante participants ont pris part à une étude portant sur un jeu de stratégie. Chacun d'eux s'adonnait à un jeu de cartes en trois temps qui ne lui était pas familier. Chacun devait utiliser une stratégie particulière de jeu soit une fois, soit trois fois. On s'attendait à ce que les participants ayant utilisé la stratégie trois fois de suite montreraient une plus forte résistance à la persuasion que ceux qui l'auraient utilisée une seule fois ou pas du tout. On a donné à la moitié des participants un message remettant en cause cette stratégie, et à l'autre moitié, un message aucunement relié à cette même stratégie. Kiesler et Mathog s'intéressaient à l'acceptation de ce message. Les résultats obtenus confirmaient leurs attentes : les participants ayant employé trois fois la même stratégie (trois actes consonants de suite) ont témoigné d'une plus grande résistance au message et ont jugé plus favorablement la stratégie que ceux qui l'avaient utilisée une seule fois. Aussi la répétition de l'acte est-elle un facteur engageant susceptible d'augmenter la résistance à une tentative de persuasion ultérieure. Selon Kiesler (1971), l'engagement peut se cumuler par la répétition des actes et l'engagement dans un acte affecte l'organisation ou la structure des cognitions liées à cet acte. La structure cognitive des participants engagés serait ainsi fortement organisée et, par conséquent, résistante à toute attaque persuasive. Salancik et Kiesler (1971) ont montré que des participants fortement engagés retiennent plus de paires de mots en accord avec leur attitude et moins de paires de mots en désaccord avec celle-ci en comparaison à des participants faiblement engagés.

\section{Hypothèses}


Partant du principe selon lequel un fort engagement, manipulé par la répétition ou la fréquence du comportement de consommation, renforce la résistance au changement de la cognition associée au comportement qu'est la consommation d'alcool :

\section{Hypothèse 1}

Les participants " buveurs habituels » (fortement engagés dans la consommation d'alcool) résisteraient davantage à une tentative de persuasion anti-alcool que les participants "buveurs occasionnels" (faiblement engagés dans la consommation d'alcool). On s'attend à ce que les buveurs occasionnels expriment une attitude moins favorable envers l'alcool de même qu'une plus forte intention de réduire leur consommation que les buveurs habituels.

\section{Hypothèse 2}

Les buveurs occasionnels, faiblement engagés dans leur consommation d'alcool, devraient se laisser persuader le plus aisément lorsque le message présente une menace forte et une grande efficacité. En effet, selon Witte (1998), les menaces de forte intensité produisent un effet sur les attitudes ou les comportements uniquement quand elles sont accompagnées d'une efficacité d'intensité équivalente. On s'attend donc à observer, chez ces buveurs occasionnels, l'attitude la moins favorable envers l'alcool et la plus forte intention de diminuer sa consommation d'alcool.

\section{Méthode}

\section{Participants}

Nous avons recruté 225 participants volontaires à l'Université de Franche-Comté à Besançon (en France). Huit questionnaires ont été éliminés pour non-respect des consignes. La passation, qui a été effectuée pendant une séance de travaux dirigés, a duré environ 15 minutes. L'étude a été présentée aux participants comme étant la première étape d'une étude de marketing social destinée à concevoir des messages de protection face a'un danger réel. On a demandé aux participants de lire le message et de répondre aux questions qui suivaient aussi franchement et honnêtement que possible. L'anonymat a été assuré.

\section{Variables indépendantes et procédure}

Le plan factoriel comprenait trois variables indépendantes croisées : le degré de menace (faible par opp. à fort), le degré d'engagement (buveurs occasionnels : engagement faible; buveurs habituels : engagement fort), le degré d'efficacité (aucun, faible, fort). Six messages ont été conçus. Chaque participant lisait un seul de ces six messages. Après la lecture, on leur demandait de remplir un questionnaire contenant les variables dépendantes. Ils devaient indiquer leur consommation d'alcool en donnant le nombre approximatif de verres qu'ils buvaient par semaine (week-end compris). Le calcul de la médiane, sur ces données, nous a permis de répartir les buveurs en deux sous-populations : ceux buvant moins de quatre verres par semaine (buveurs occasionnels : engagement faible); ceux buvant plus de 
quatre verres par semaine (buveurs habituels : engagement fort). Notre médiane (quatre verres par semaine) se rapproche de celle communément indiquée dans la documentation. Par exemple, Boscher (2002) avait réparti ses participants, buveurs d'alcool, en petits et en grands consommateurs au moyen d'une valeur médiane de sept verres par semaine.

Tous les messages présentés comprenaient deux parties. La première manipulait la description de la menace; la seconde, l'efficacité des recommandations. Chacune de ces parties a été prétestée. Un autre prétest a été réalisé afin de s'assurer de l'obtention effective de deux niveaux de peur et de deux niveaux d'efficacité. On a donné à deux groupes de 30 participants la première partie du message " menace ", conçue précédemment. Un groupe a lu le message fortement menaçant, l'autre, le message faiblement menaçant. Les participants se sont ensuite positionnés, à l'aide d'échelles, selon quatre adjectifs ayant trait aux affects et traduisant la peur (" effrayé », " relax ", " calme », " inquiet »). Ces échelles constituaient un index mesurant la peur. Les résultats ont montré que cette partie du message a efficacement crée deux niveaux de peur (faible et fort) chez les deux groupes de participants - $[\mathrm{t}(58)$ 2,87; p. 0,04 : faible ( $M$ 4,2; SD 1,54) et fort (M 6,$7 ;$ SD 2,30)]. La même procédure s'appliquait à la seconde partie du message. Deux groupes de 25 participants ont lu le message fortement efficace; un autre groupe, le message faiblement efficace. Les participants ont ensuite donné leur avis sur le degré d'efficacité des recommandations proposées dans le texte selon une échelle de 10 points.

Les résultats ont montré que cette partie du message a efficacement crée deux niveaux d'efficacité (faible et fort) chez les deux groupes de participants - [t(48) 2,36; p. 0,05: faible (M 3,4, SD 1,78) et fort (M 5,4; SD 1,55)].

Instruments de mesure

Après la lecture du message, les participants ont rempli d'abord une échelle mesurant la peur éprouvée. La peur est une émotion évaluée négativement, accompagnée d’un fort niveau d'éveil. Rogers (1983) a montré que la peur peut être associée à une évaluation cognitive. En effet, l'évaluation d'adjectifs présentés sous forme d'échelles, comme « nerveux ", " apeuré», " inquiet ", " tendu ", " effrayé ", " anxieux », corrèlent positivement avec les mesures physiologiques de l'éveil de la peur. Ainsi, les participants devaient se positionner sur ces échelles allant de 0 (pas du tout) à 10 (tout à fait) $(-0,88)$. Les participants devaient ensuite exprimer leur attitude envers l'alcool à l'aide de quatre échelles en 10 points, allant de 0 à 10 ("Selon vous, l'alcool est-il quelque chose de : désagréable/ agréable, mauvais/bon, négatif/positif, pas nécessaire/nécessaire $»)-(-0,807)$. Finalement, on a demandé aux participants s'ils avaient l'intention de réduire leur consommation d'alcool sur une échelle en 10 points, allant de 1 (pas du tout) à 10 (tout à fait).

\section{Mesures annexes}


Les participants devaient dire s'ils consommaient de l'alcool (oui ou non). Si oui, ils devaient indiquer combien de verres, en moyenne, ils consommaient par semaine. Nous avons effectué une analyse de covariance (ANCOVA). Les participants devaient indiquer leur âge et leur sexe, puis indiquer selon une série d'échelles en 10 points leur perception des aspects suivants : leur état de santé; s'ils avaient déjà adopté des comportements de protection face à l'alcool; s'ils avaient déjà souffert d'un problème lié à l'alcool; leurs connaissances sur les effets de l'alcool et les moyens de s'en prémunir, et s'ils connaissaient quelqu'un ayant souffert des effets de l'alcool. L'analyse de covariance n'a révélé aucun effet de ces variables sur nos manipulations expérimentales. Elles n'ont eu aucun effet sur la peur, l'efficacité et la consommation d'alcool. Les variables manipulées n'ont pas influé sur la perception de la quantité d'information ni sur la crédibilité du message. En dernier lieu, on a demandé aux participants s'ils soupçonnaient quelque chose sur le déroulement de l'expérience. Ils ont ensuite été démystifiés, puis remerciés.

\section{Résultats}

Nous avons utilisé pour le traitement de nos résultats et pour chacune de nos variables dépendantes l'analyse de variance (ANOVA). Une analyse de médiation par régression multiple a ensuite été effectuée sur ces variables dépendantes (peur, attitude et intention comportementale).

Nous avons commencé l'analyse par une vérification de la bonne manipulation de la variable indépendante " menace " sur la variable dépendante "peur ». Nous avons compilé les six scores de chaque participant pour établir un index de la peur, puis l'ensemble des scores a été standardisé. Après vérification de l'homogénéité des variances, nous avons réalisé une ANOVA sur nos trois variables indépendantes. II s'agissait d'un plan factoriel 2 (menace : faible par opp. 2 (engagement : faible par opp. à fort) X 3 (efficacité : aucune ou faible ou forte). Nous avons, pour la suite de l'analyse statistique, utilisé le test de NewmanKeuls, lequel permet de comparer entre elles les moyennes des conditions expérimentales (Howell, 1998). Les résultats sont consignés dans le Tableau 1. Les résultats obtenus font état d'une bonne manipulation de la variable " menace ". Ils montrent un effet principal de cette variable $[F(1,215) \quad 8,293 ; p$ 0,005]. Les moyennes et les écarts types de cet effet principal montrent que les participants placés en situation de forte menace éprouvent plus de peur $(M 0,179 ; S D$ 1,038) que ceux qui sont placés en situation de faible menace ( $M$ 0,196; SD 0,736). On observe aussi un effet principal marginalement significatif de l'efficacité $[F(2,215) 2,927 ; p \quad 0,06]$. Les moyennes et les écarts types de cet effet principal montrent que les participants placés en situation de forte efficacité ( $M 0,167$; SD 1,079) éprouvent tendanciellement plus de peur que ceux qui sont placés en situation de faible efficacité (M 0,22; SD 0,693). On n'a constaté aucun effet de la variable « consommation ». 
Peur moyenne comme fonction du degré d'engagement, de la menace et de l'efficacité. Plus les chiffres sont élevés, plus les participants éprouvent de la peur (scores standardisés). Les écarts types figurent entre parenthèses

\begin{tabular}{|c|c|c|c|c|c|c|}
\hline & \multicolumn{3}{|c|}{$\begin{array}{l}\text { Engagement fort } \\
\text { Buveurs réguliers }\end{array}$} & \multicolumn{3}{|c|}{$\begin{array}{l}\text { Engagement faible } \\
\text { Buveurs occasionnels }\end{array}$} \\
\hline & \multicolumn{3}{|c|}{ Efficacité $^{* *}$} & \multicolumn{3}{|c|}{ Efficacité ${ }^{* *}$} \\
\hline & Sans & Faible & Forte & Sans & Faible & Forte \\
\hline Faible menace & $\begin{array}{c}0,071(1,171) \\
\mathrm{n}=22\end{array}$ & $\begin{array}{c}-0,198(0,871) \\
n=18\end{array}$ & $\begin{array}{c}-0,413(0,464) \\
\mathrm{n}=17\end{array}$ & $\begin{array}{c}-0,374(0,4) \\
\mathrm{n}=19\end{array}$ & $\begin{array}{c}-0,309(0,602) \\
n=19\end{array}$ & $\begin{array}{c}0,051(0,909) \\
\mathrm{n}=17\end{array}$ \\
\hline Forte menace & $\begin{array}{c}0,233(1,145) \\
\mathrm{n}=24\end{array}$ & $\begin{array}{c}-0,137(0,567) \\
\mathrm{n}=21\end{array}$ & $\begin{array}{c}0,346(1,503) \\
\mathrm{n}=21\end{array}$ & $\begin{array}{c}0,184(0,839) \\
\mathrm{n}=17\end{array}$ & $\begin{array}{c}-0,236(0,734) \\
\mathrm{n}=15\end{array}$ & $\begin{array}{c}0,685(1,44) \\
n=17\end{array}$ \\
\hline
\end{tabular}

Nota. Les modalités des variables partageant le même chiffre differe soit significativement $(\mathrm{p}<0,05)$, soit marginalement $(* \mathrm{p}<0,10)$.

Il apparaît que les deux variables " menace " et " efficacité " agissent indépendamment l'une de l'autre sur la variable dépendante "peur » et que l'action combinée de ces deux variables relève de la somme de l'action distincte de chacune d'entre elles (effets additifs sur la variable " peur »). En effet, nos résultats montrent, sans tenir compte de la variable "engagement ", que le plus fort niveau de peur (M 0,515; SD 1,471) s'observe dans les situations de forte menace et de forte efficacité prises ensemble. La moyenne des deux conditions - forte menace et forte efficacité -, prises ensemble et sans tenir compte de la variable " engagement ", diffère significativement de la moyenne des deux conditions forte menace et faible efficacité prises ensemble ( $M 0,1865$; SD 0,650); $p 0,02$, test de NewmanKeuls) et de la moyenne des deux conditions faible menace et forte efficacité prises ensemble ( $M$ 0,18; SD0,686); p0,03, test de Newman-Keuls). Les résultats obtenus ne suivent pas le modèle de Witte. Selon ce modèle, on s'attendrait à observer la peur la plus forte lorsque la menace est forte et l'efficacité faible. Toutefois, Witte (1992, p. 344) suggère que des individus peuvent éprouver de la peur lorsqu'ils ont emprunté la voie de le contrôle du danger (c.-à-d., forte menace, forte efficacité). Ce niveau de peur peut produire un effet de résistance à la persuasion sur les variables attitude, intention et comportement. En effet, selon Witte, la résistance à la persuasion s'observe lorsque la peur est éveillée chez les individus. La peur est une cause directe de la résistance : l'évitement des recommandations proposées et l'adoption d'une attitude contraire à celle qui est défendue dans le message. Tout porte donc à croire, après ce premier traitement et contrairement à notre hypothèse 2 , que les participants placés dans les conditions de forte menace et forte efficacité vont adopter une attitude contraire à celle qui est défendue dans le message.

\section{1- La variable dépendante " attitude »}

Nous avons compilé les quatre scores de chaque participant afin de construire un index d'attitude, puis l'ensemble des scores a été standardisé. Après vérification de l'homogénéité des variances, nous avons réalisé une analyse de variance (ANOVA) sur nos trois variables indépendantes selon le plan factoriel (menace : faible par opp. A forte) $X$ (engagement : faible par opp. A (efficacité : aucune vs faible par opp. à forte). Pour la suite de l'analyse statistique, nous avons utilisé le test de Newman-Keuls. Les résultats sont consignés dans le Tableau 2. 
Attitude moyenne envers l'alcool comme fonction du degré d'engagement, de la menace et de l'efficacité. Plus les chiffres sont élevés, plus les participants ont une attitude favorable envers l'alcool (scores standardisés). Les écarts types figurent entre parenthèses

\begin{tabular}{|c|c|c|c|c|c|c|}
\hline & \multicolumn{3}{|c|}{$\begin{array}{l}\text { Fort engagement } \\
\text { Buveurs réguliers }\end{array}$} & \multicolumn{3}{|c|}{$\begin{array}{c}\text { Faible engagement } \\
\text { Buveurs occasionnels }\end{array}$} \\
\hline & \multicolumn{3}{|c|}{ Efficacité } & \multicolumn{3}{|c|}{ Efficacité } \\
\hline & Sans & Faible & Forte & Sans & Faible & Forte \\
\hline Faible menace & $\begin{array}{l}-0,205(0,867) \\
-0,086(0.770)\end{array}$ & $-0,147(0,698)$ & $-0,043(0,986)$ & $0,381(1,03)$ & $0,070(0,678)$ & $0,498(1,021)$ \\
\hline
\end{tabular}

Nota. Les modalités des variables partageant le même chiffre differe soit significativement $(\mathrm{p}<0,05)$, soit marginalement $\left({ }^{* *} \mathrm{p}<0,10\right)$.

Les résultats obtenus montrent un effet principal de la consommation $[F(1,215) \quad 12,947 ; p$ $0,001]$. Les moyennes et les écarts-types de cet effet principal montrent que les participants fortement engagés (buveurs habituels) ont une attitude beaucoup moins favorable envers l'alcool ( $M \quad$ 0,214; SD 0,906) que ceux qui sont faiblement engagés ( $M \quad 0,247$; SD 0,916). On observe une interaction significative entre la variable " engagement» et la variable «efficacité » $[F(2,215)$ 4,06; p 0,02]. Les résultats sont consignés dans le Tableau 3.

Tableau 3

Attitude moyenne envers l'alcool comme fonction du degré d'engagement et de l'efficacité. Plus les chiffres sont élevés, plus les participants ont une attitude favorable envers l'alcool (scores standardisés). Les écarts types figurent entre parenthèses

\begin{tabular}{lccc}
\hline & \multicolumn{3}{c}{ Efficacité } \\
\cline { 2 - 4 } & Sans & Faible & Forte \\
\hline Engagement faible & 0,309 & $-0,153$ & 0,585 \\
Buveurs occasionnels & $(0,819)$ & $(0,956)$ & $(1,098)$ \\
Engagement fort & $-0,145$ & $-0,162$ & $-0,335$ \\
Buveurs réguliers & $(0,819)$ & $(0,902)$ & $(0,999)$ \\
\hline
\end{tabular}

Parmi les participants ayant lu le message sans la composante "efficacité", ceux qui sont faiblement engagés ont une attitude significativement plus favorable envers l'alcool $(M$ $0,309)$ que ceux qui sont fortement engagés $\left(M_{-}{ }_{-} 0,145\right) ; p_{-} 0,04$, selon le test de Newman-Keuls. Parmi les participants ayant lu le message avec une composante forte efficacité, ceux qui sont faiblement engagés ont une attitude beaucoup plus favorable envers l'alcool $\left(M_{-} 0,585\right)$ que ceux qui sont fortement engagés $\left(M_{-} 0,335\right) ; p_{-} 0,001$, selon le test de Newman-Keuls.

\section{2 - La variable dépendante « intention»}

Nous avons utilisé la même procédure statistique que précédemment. Après avoir vérifié l'homogénéité des variances, nous avons réalisé une ANOVA sur nos trois variables indépendantes) selon le plan factoriel présenté précédemment. Les résultats en sont consignés dans le Tableau 4. 
Intention moyenne envers l'alcool comme fonction du degré d'engagement, de la menace et de l'efficacité. Plus les chiffres sont élevés, plus les participants ont l'intention de réduire leur consommation d'alcool (scores standardisés). Les écarts types figurent entre parenthèses

\begin{tabular}{|c|c|c|c|c|c|c|}
\hline & \multicolumn{3}{|c|}{ Fort engagement } & \multicolumn{3}{|c|}{ Faible engagement } \\
\hline Faible menace & $-0,006(0,895)$ & $0,314(1,071)$ & $-0,250(0,744)$ & $0,107(1,061)$ & $-0,287(0,732)$ & $-0,139(0,834)$ \\
\hline Forte menace & $-0,228(0,836)$ & $-0,210(0,975)$ & $0,665(1,550)$ & $-0,051(0,819)$ & $-0,053(1,061)$ & $0,125(0,885)$ \\
\hline
\end{tabular}

On observe une interaction entre la variable " menace» et la variable " efficacité " $[F(2$, 215) _ 3,$\left.6175 ; p_{-} 0,03\right]$. Aucune autre différence significative n'est relevée. Les résultats de cette interaction sont consignés dans le Tableau 5.

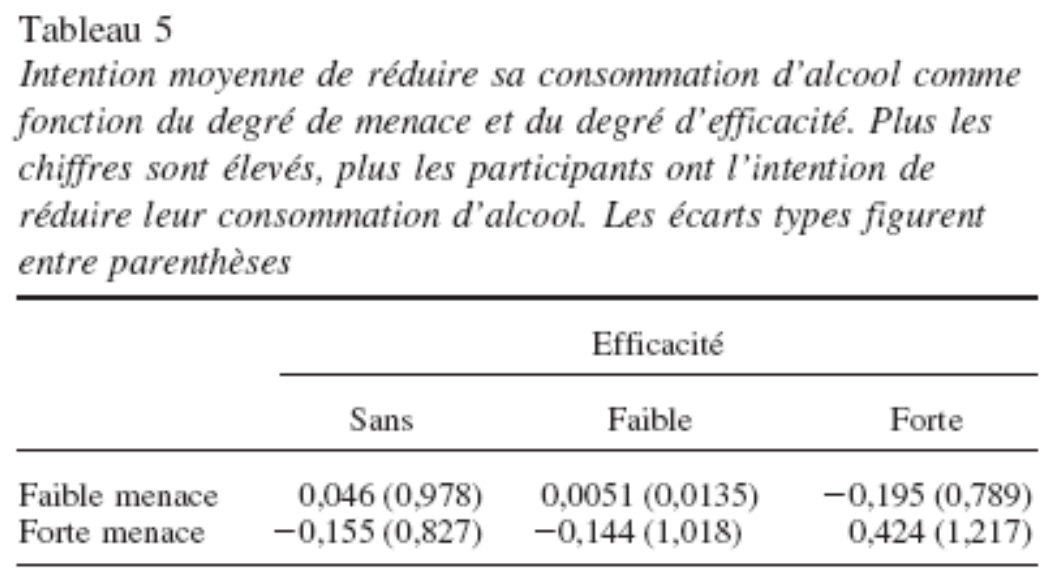

Les résultats montrent que, parmi les participants ayant lu le message incluant une forte efficacité, ceux ayant reçu une forte menace ont significativement plus l'intention de réduire leur consommation d'alcool $\left(M_{-} 0,424\right)$ que ceux ayant reçu une faible menace $\left(M_{-}\right.$ _0,195); $p_{-} 0,01$, test de Newman-Keuls). Par ailleurs, l'intention moyenne des participants placés en situation de forte menace et de forte efficacité $\left(M_{-} 0,424\right)$ diffère beaucoup de l'intention moyenne des participants placés en situation de forte menace et de faible efficacité $\left(M_{-} 0,144\right)$ et de forte menace et sans efficacité $\left(M_{-}{ }_{-}, 155\right) ; p_{-} 0,03$ et $p_{-} 0,03$, respectivement, selon le test Newman-Keuls. Pour cette variable dépendante, les résultats obtenus ne concordent pas avec nos hypothèses 1 et 2 . Aucun effet de l'engagement (faible par opp. à fort) n'est observé par analyse de variance. Afin d'établir une éventuelle relation entre la peur, l'attitude et l'intention, nous avons réalisé des analyses de médiation. Nous désirions déterminer si l'attitude $(M)$ pouvait entièrement médiatisé l'effet de la peur $(X)$ sur I'intention $(\mathrm{Y})$.

Selon Baron et Kenny (1986), Brauer (2000) puis Judd (2000), pour déterminer un médiateur $M$ de l'effet d'une variable $X$ sur une autre variable $Y$ dans un plan intrasujets, quatre conditions doivent être remplies : 
1) la variable $X$ doit avoir un effet sur la variable $Y$;

2) la variable $X$ doit avoir un effet sur le médiateur $M$;

3) le médiateur $M$ doit avoir un effet sur la variable $Y$ si l'on contrôle statistiquement l'effet de la variable $X$ sur la variable $Y$;

4) I'effet de la variable $X$ sur la variable $Y$ doit disparaître si l'on contrôle statistiquement l'effet du médiateur $M$ sur la variable $Y$.

Afin de mettre à l'essai le modèle selon lequel l'attitude médiatiserait l'effet de la peur sur l'intention, nous avons testé chacune de ces étapes à l'aide de régressions simples et multiples (voir Brauer, 2000). Notre première analyse en piste, appliquée à nos résultats (buveurs habituels et occasionnels confondus), montre que les trois premières conditions sont remplies :

1) la variable " peur » a un effet sur la variable « intention " ${ }_{-}-0,135, t(225) \_2,036 ; p_{-}$ $0,05)$;

2) la variable " peur » a un effet sur la variable " attitude " ${ }_{-}-0,206, t(225) \_3,157 ; p_{-}$ $0,002)$;

3) la variable médiatrice " attitude " a un effet sur la variable " intention " si l'on contrôle statistiquement l'effet de la variable " peur » sur la variable « intention » ${ }_{-}{ }_{-} 0,19, t(224)$ _2,915; $p_{\text {_ }}$ 0,004). La valeur bêta de l'effet de la variable " attitude " sur la variable " intention » est significativement différente de zéro : cette troisième condition est remplie;

4) cette condition n'est pas remplie. L'effet de la variable variable " peur » sur la variable " intention " ne disparaîtpas si l'on contrôle l'effet de la variable médiatrice variable " attitude " sur la variable " intention " $\left(-0,174 ; t(224) \_2,626 ; p_{-} 0,01\right)$. Signalons que le bêta obtenu dans notre modèle entre " peur " et variable " attitude " est très proche des valeurs observées par Boster et Mongeau (1984) : 0,21; Mongeau (1998) : 0,20; Witte et Allen (2000) : 0,15. Enfin, Witte et Allen (2000) rapportent une valeur égale a` 0,126 ( $N_{-}$ 9686) entre " peur » et " intention ».

Selon le même principe que celui qui a été utilisé précédemment, nous avons réalisé deux autres analyses de médiation. La première analyse concernait uniquement les buveurs habituels, la seconde, les buveurs occasionnels. Nous désirions déterminer si l'effet de la peur $(X)$ sur l'intention $(Y)$ est médiatisé complètement par l'attitude $(M)$. Notre première analyse, appliquée à nos résultats, concernait les buveurs habituels d'alcool. La variable " peur » ne produit pas d'effet sur la variable « intention " $\left(\ldots 0,12, t(121) \_1.344 ; p_{\_} 0,19\right) \mathrm{ni}$ sur la variable " attitude " $\left(_{-}-0,11, t(121) \_1,219 ; p_{-} 0,23\right)$. Signalons, enfin, que la variable " attitude " produit un effet inverse sur la variable " intention " $(0,20$, $t(120) \_2,254 ; p_{-}$0,03). Certains _ n'étant pas significatifs à ce stade de l'analyse, nous ne pouvons conclure à une relation. Pour les buveurs habituels d'alcool, seule l' « attitude » est corrélée négativement et significativement à $l^{\prime}$ " intention " $\left(r_{-}-0,20 ; p_{-} 0,05\right)$. Ces résultats, par analyse de médiation, correspondent à nos attentes : un fort engagement " freine " ou " casse " toute tentative de persuasion ultérieure par la peur. En ce qui concerne les buveurs occasionnels d'alcool, la variable " peur " ne produit pas d'effet sur la variable " intention " $\left(_{-}-0,155, t(102){ }_{-} 1,588 ; p_{-} 0,12\right)$, mais a un effet sur la variable " attitude " (condition 2, _ $0,34, t(102) \_3,645 ; p_{-} 0,001$ ). Signalons aussi (condition 3 ) que la variable " attitude » produit un effet tendanciel sur la variable « intention » 
_ $\left.0,19, t(102) \_\_1,799 ; p \_0,08\right)$ si l'on contrôle statistiquement l'effet de la variable " peur » sur la variable " intention ». De façon plus importante, l'effet de la variable " peur » sur la variable " intention "se manifeste $\left(_{-}-0,218, t(101){ }_{-} 2,121 ; p_{-} 0,04\right)$ lorsqu'on contrôle l'effet de la variable " attitude " sur la variable " intention ". On ne peut en conclure pour autant à une relation complète ou partielle entre ces trois variables : les conditions 1,3 et 4 ne sont pas correctement remplies.

\section{Discussion}

Cette recherche avait pour objectif de tester expérimentalement les effets d'un message anti-alcool, faisant appel à la peur, sur des participants faiblement engagés dans leur consommation comparativement à des participants fortement engagés dans leur consommation.

Premièrement, comme nous nous y attendions, les participants fortement engagés (buveurs habituels), comparativement aux faiblement engagés (buveurs occasionnels), ont résisté à la tentative de persuasion. Cette résistance, comme fonction du degré d'engagement (faible par opp. A fort), s'observait dans leur attitude envers l'alcool.

Deuxièmement, contrairement à notre première hypothèse, les participants faiblement engagés (buveurs occasionnels) ont adopté une attitude plus favorable envers l'alcool que ceux fortement engagés (buveurs habituels) lorsqu'ils ont été placés en condition de forte efficacité, peu importe le degré de menace (faible ou fort).Nous suggérons que la vulnérabilité perçue a un effet. Si l'individu ne se perçoit pas comme étant vulnérable, il n'adhérera pas aux recommandations proposées dans le message (Girandola, 2000a).Les buveurs occasionnels (faiblement engagés) ne se percevraient pas comme étant vulnérables et, par conséquent, exprimeraient une attitude plus favorable envers l'alcool que les buveurs habituels (fortement engagés). Notre première hypothèse trouve une validation lorsqu'on s'intéresse aux effets de la peur sur l'intention. On observe un effet de la peur sur l'intention comportementale des participants faiblement engagés (buveurs occasionnels). Plusieurs

bêtas obtenus dans les analyses de médiation pour les buveurs occasionnels sont plus forts que ceux qui ont été obtenus pour les buveurs habituels. Nous pouvons, par conséquent, suggérer que la peur a un plus grand effet sur l'intention comportementale des buveurs occasionnels que sur celle des buveurs habituels. Même si l'on ne constate pas de relation explicite, nous avons constaté que, pour les buveurs occasionnels, l'effet de la " peur " sur l'« intention " apparaît si l'on contrôle statistiquement l'effet de l'« attitude» sur I'«intention» .

Troisièmement, contrairement aux attentes, la variable " engagement " n'a pas d'effet important sur l'intention comportementale. Nos résultats sont compatibles avec le Modèle des processus parallèles étendus de Witte (1998) : la plus forte intention de réduire la consommation d'alcool s'obtient dans la condition forte menace et forte efficacité. Witte (1992) a obtenu, chez des adolescents, la plus forte intention comportementale d'utiliser des préservatifs dans la situation forte menace et forte efficacité. Parallèlement, c'est dans la condition de forte menace et de forte efficacité que l'on observe la plus forte intention de réduire sa consommation d'alcool. Souvenons-nous aussi que c'est dans cette condition expérimentale que les participants éprouvent le plus de peur. Ce dernier résultat montre qu'une forte menace associée à une forte efficacité agit efficacement sur l'intention comportementale des buveurs. Ces résultats nous incitent à prendre en compte les recommandations concernant la conception des campagnes de prévention. Selon Witte et Allen (2000), les campagnes de prévention devraient accentuer la menace et, tout en 
renforçant l'efficacité perçue (efficacité des recommandations et autoefficacité) afin que les participants adhèrent aux recommandations proposées. Ces chercheurs ont montré que la menace de forte intensité est efficace uniquement quand elle s'accompagne d'une efficacité d'une intensité équivalente. D'un point de vue appliqué, Witte, Cameron, McKeon et Berkowitz (1996), puis Witte, Meyer et Martell (2001) ont validé une échelle de diagnostic sur les comportements à risque. Elle permet d'établir rapidement le degré de croyance en la menace et dans l'efficacité. Ce repérage initial permet de sélectionner, parmi plusieurs messages déjà rédigés, celui dont les effets seront optimaux parmi le public concerné. Autrement dit, le modèle de Witte représente un réel progrès dans la compréhension des mécanismes cognitifs induisant la peur et le changement comportemental.

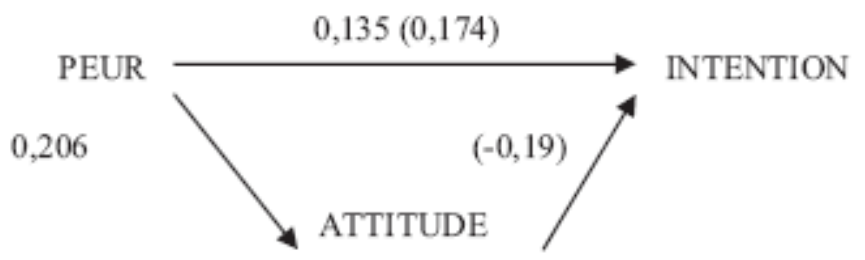

Figure 1. Modèle en pistes. La peur et l'attitude sont deux prédicteurs directs de l'intention. Les valeurs sans les parenthèses correspondent au coefficient bêta indiquant la relation entre deux variables. Les valeurs avec les parenthèses indiquent la relation lorsqu'une troisième variable est contrôlée statistiquement. Acceptation du message comme fonction du degré de menace perçue et de l'efficacité perçue (selon Witte, 1994).

Quatrièmement, les résultats obtenus par analyse de régression (voir la Figure 1) montrent que la peur et l'attitude sont des prédicteurs importants et indépendants de l'intention. Autrement dit, la peur permet de prédire relativement bien l'intention, quelle que soit l'attitude des participants. En revanche, la valeur de l'attitude permet de prédire relativement bien, mais en sens inverse, l'intention des participants, quel que soit le niveau de peur exprimé par ces derniers. L'attitude est un prédicteur de l'intention : la corrélation, bien que significative, s'avère négative. Notre modèle ne laisse pas paraître de médiation complète où la totalité de l'effet de la variable " peur " sur la variable " intention " est médiatisée par la variable " attitude ". Selon Brauer (2000), une variable peut médiatiser partiellement la relation entre deux autres variables. Autrement dit, si les conditions 1, 2 et 3 sont satisfaites et si la condition 4 ne l'est pas (voir la section sur les résultats), nous pouvons en conclure que les données sont concordantes avec un modèle de médiation partielle. Il se peut, par exemple, que l'effet de la "peur » sur l'« intention "soit médiatisé par deux variables dont une seule ( $I$ " ' attitude ") a été incluse dans notre recherche. Nous suggérons la "menace perçue " comme seconde variable médiatrice. Les résultats de quelques recherches abondent dans ce sens. Par exemple, Rippetoe et Rogers (1987) ont montré que la peur et la menace perçue sont étroitement associées. Qui plus est, Rogers et Mewborn (1976) ont montré que la relation entre la peur et l'intention disparaissait lorsqu'on contrôlait statistiquement la sévérité perçue, une composante de la " menace perçue ". Selon notre analyse en pistes, la peur ne permet pas de prédire directement les intentions. Tout porte à croire que la peur peut produire des changements dans les intentions, voire les comportements, lorsqu'elle est médiatisée par la menace perçue, à tout le moins en situation de forte efficacité. 
Nous devons signaler quelques limites de notre étude. La première est l'absence d'un groupe contrôle. Nous n'avons pas mesuré l'attitude et l'intention comportementale chez les buveurs qui ne lisent aucun message. II se peut que les individus possédant une attitude et une intention (positive ou négative) envers l'alcool prêtent moins attention au message ou, à l'inverse, leur conviction est renforcée. Selon nous, les participants n'avaient pas d'attitude bien définie sur ce point : l'analyse de covariance effectuée dans cette expérience ne montre pas l'influence de la connaissance des effets de l'alcool sur les variables mesurées. Une deuxième limite concerne la répartition des participants buveurs d'alcool dans les catégories " occasionnels " et " habituels ". Cette répartition a été effectuée selon leur propre évaluation de leur consommation. Malgré les précautions méthodologiques qui ont été prises, il se peut que ces auto-évaluations aient subi l'effet de l'attitude initiale des participants envers l'alcool. Eagly, Chen, Chaiken et Shaw-Barnes (1999) ont montré que l'attitude initiale des individus envers un objet produit un impact sur la mémoire des événements liée à cet objet. On peut penser que les participants ayant une attitude positive à l'égard de l'alcool ont tendance à sous-estimer leur consommation, alors que ceux qui se soucient plus de leur santé ont tendance à la surestimer. Par ailleurs, les deux groupes (faible engagement et fort engagement) diffèrent peu quant à leur consommation d'alcool (cinq verres par semaine pour les buveurs habituels, trois verres pour les buveurs occasionnels), ce qui peut réduire l'impact de la variable engagement sur les variables dépendantes. La troisième limite est l'ordre de passation des variables dépendantes. Nous avons évalué la peur après la lecture du message, mais avant l'évaluation de l'attitude envers l'alcool. Cet ordre de passation, semblable dans toutes les conditions expérimentales, pourrait avoir mis en évidence la peur provoquée par la menace ainsi que les dangers de l'alcool et, par conséquent, avoir réduit l'impact des autres facteurs manipulés. II appert qu'un rééquilibre des différentes variables dépendantes serait nécessaire dans le cadre d'une prochaine étude afin de s'assurer de la mesure efficace de leurs effets.

Une autre limite porte sur la mesure effective de l'intention et du comportement. Dans cette expérience, nous avons évalué l'effet des messages sur l'attitude et l'intention comportementale à l'aide d'échelles. Nous n'avons pas de mesure du comportement effectif ou d'évocation libre de ce comportement. L'ajout de ces composantes permettrait de vérifier l'effet véritable de la peur sur les comportements. La portée de la recherche s'en trouverait ainsi élargie. Nous n'avons pas mesuré l'effet des différences individuelles sur la persuasion. On sait, par exemple, que les individus " répresseurs " nient plus facilement l'information qui suscite des craintes que les individus " sensitifs" (Epstein \& Fenz, 1967). On sait aussi que des individus ayant un fort besoin de cognition, c'est-à-dire une appétence pour l'effort cognitif, adhèrent plus facilement aux recommandations proposées en réaction au message menaçant que ceux ayant une faible besoin de cognition (Ruiter, Verplanken, De Cremer \& Kok, 2004). Selon nous, le fait de ne pas avoir pris en compte certains éléments caractéristiques des buveurs occasionnels et des buveurs habituels constitue une autre limite de l'expérience. Ces éléments auraient permis une analyse plus précise des processus à l'oeuvre dans une tentative de persuasion par la peur (Boscher, 2002).

Enfin, l'échantillon utilisé comporte lui aussi une limite. Etant constitué uniquement d'étudiants, il restreint la portée de nos conclusions. Un test dans le grand public permettrait d'évaluer dans quelle mesure les individus exposés à des messages de cette nature réduisent effectivement leur consommation d'alcool. 


\section{Résistance et communication engageante}

Les résultats mettent en question le lien entre l'engagement et la résistance à un message. En effet, selon Kiesler (1971), puis Joule et Beauvois (1998), l'engagement est source de résistance du fait qu'il renforce la cognition représentant le comportement à changer (ici, la consommation d'alcool). Nous adhérons à cette vision de la résistance. Toutefois, une autre perspective mérite, selon nous, d'être élaborée à la lumière des travaux sur l'engagement. Nous proposons que l'engagement dans un comportement participe a' définir la force de l'attitude qui lui est conforme. Selon Petty et Krosnick (1995), les attitudes fortes possèdent quatre caractéristiques : elles résistent au changement, elles sont stables dans le temps, elles ont des répercussions sur les processus cognitifs et elles déterminent le comportement social. La force d'une attitude, dans les théories de la persuasion, est évaluée en fonction de l'ampleur des mécanismes cognitifs, tels le jugement ou l'évaluation, déployés lors du traitement de l'information persuasive. Le raisonnement, pour expliquer la résistance, pour ce qui a trait à la cognition la plus résistante au changement ou à la force de l'attitude, s'inscrit finalement dans une problématique liant à la fois trois cadres théoriques: engagement, exposition à l'information (Frey, 1986) et persuasion par la peur (Chen \& Chaiken, 1999; Petty \& Cacioppo, 1986; Petty \& Wegener, 1999). Nous avons récemment insisté sur l'intérêt à travailler sur ces théories jusqu'à maintenant disjointes et avons mis en relief les effets de l'engagement sur l'exposition à l'information et sur les tentatives de persuasion (Girandola, 2003). Le paradigme de la communication engageante se propose de faire le lien entre les travaux sur l'engagement, l'exposition à l'information et la persuasion.

Pour ces travaux, qu'ils soient fondamentaux (Girandola, Joule \& Didelot, 2002) ou appliqués (par ex., Joule, Girandola \& Bernard, 2007), la question ne se résume pas seulement à «Qui dit quoi, à qui, dans quel canal et avec quel effet ? " (Laswell, 1948, p. 37) La question réside en fait dans la communication engageante : quels sont les actes préparatoires engageants ou soubassements comportementaux qui influent sur la sélection de l'information ou sur la persuasion? Le succès et la réussite des tentatives de persuasion peuvent être analysés en fonction de la connaissance de ces soubassements comportementaux. C'est le cas, dans notre étude, de l'engagement dans la consommation d'alcool. Cette nouvelle perspective pourrait constituer une solution de rechange au modèle clinique de l'entretien motivationnel chez les buveurs d'alcool (McNally, Palfai \& Kahler, 2005; Miller \& Rollnick, 2002). On peut aussi s'interroger sur les actes préparatoires ou soubassements comportementaux engageants à obtenir de la part du récepteur pour faciliter la persuasion. Dans ce cas, selon Joule, Girandola et Bernard (2007), la question devrait se reformuler ainsi : "Qui dit quoi, à qui, dans quel canal, en lui faisant faire quoi et avec quel effet comportemental ? " L'acte préparatoire engageant doit être réalisé dans un contexte (c.-à-d., libre choix, absence de promesse de récompense et de menace de punition) où l'individu peut tisser un lien entre ce qu'il fait et ce qu'il est, et finalement entre ce qu'il est et la cause à défendre ou à promouvoir (Deschamps, Joule \& Gumy, 2005). Nos premières recherches dans ce paradigme associent engagement et persuasion anti-alcool (Michelik \& Girandola, 2004). Nous souhaitons qu'elles permettront de mieux comprendre la résistance à la persuasion. 


\section{Références}

Andrews, J. A., Tildesley, E., Hops, H., \& Li, F. (2002). The influence of peers on young adult substance use. Health Psychology, 21, 349-357.

Baron, R. M., \& Kenny, D. A. (1986). The moderator-mediator variable distinction in social psychological research: Conceptual, strategic, and statistical considerations. Journal of Personality and Social Psychology, 51, 1173-1182.

Betsch, T., \& Haberstroh, S. (2005). Current research on routine decision making: Advances and prospects. In T. Betsch., \& Haberstroh (Eds.), The routines of decision making (pp. 359376). Erlbaum: Mahwah.

Boscher, G. (2002). Les expectations d'alcool, de la théorie à l'évaluation. 4e Congrès international de psychologie sociale en langue française, 1-4 septembre (Athènes, Grèce).

Boscher, G., Pavic, G., \& Pérrissol, S. (2002). Attitude, comportements et prévention face aux conduites de consommation d'alcool. Dans C. Bonardi., F. Girandola., N. Roussiau., et N. Soubiale (Ed.), Psychologie sociale appliquée : environnement, sante', qualite' de vie (pp. 275-293). Editions In-Press.

Boster, F. J., \& Mongeau, P. (1984). Fear-arousing persuasive messages. In R. N. Bostrom., \& B. H. Westley (Eds.), Communication Yearbook 8 (pp. 330-375). Newbury Park, CA : Sage.

Brauer, M. (2000). L'identification des processus médiateurs dans la recherche en psychologie. L'Anne'e psychologique, 100, 661-681.

Brauer, M. (2002). L'analyse des variables indépendantes continues et catégorielles : alternatives à la dichotomisation. L'Année psychologique, 102, 449-484.

Cerclé, A. (2002). L'étude narratologique et stratégique des modérateurs

psycho-sociaux des ajustements post-alcooliques. Dans C. Bonardi., F. Girandola., N. Roussiau., \& N. Soubiale (Eds.), Psychologie sociale appliquée : environnement, sante', qualite' de vie (pp. 295-312).Editions In-Press.

Chassin, L., Tetzloff, C., \& Hershey, M. (1985). Self-image and social image factors in adolescent alcohol use. Journal of Studies on Alcohol, 46, 39-47.

Chen, S., \& Chaiken, S. (1999). The Heuristic-systematic model in its broader context. In S. Chaiken., \& Y. Trope (Eds.), Dual-process theories in social psychology (pp. 73-96). Guilford Press.

Crano, W. D., \& Prislin, R. (2006). Attitudes and persuasion. Annual Review of Psychology, 57, 345-374. 
Deschamps, J.-C., Joule, R.-V., \& Gumy, C. (2005). La communication engageante au service de la réduction de l'abstentionnisme électoral. Revue européenne de psychologie appliquée, 55, 21-27.

Eagly, A. H., Chen, S., Chaiken, S., \& Shaw-Barnes, K. (1999). The impact of attitudes on memory: An affair to remember. Psychological Bulletin, 125, 64-89.

Epstein, S., \& Fenz, W. D. (1967). The detection of emotional stress through variations in perceptual threshold and physiological arousal. Journal of Experimental Research in Personality, 2, 191-199.

Frey, D. (1986). Recent research on elective exposure to information. In L. Berkowitz (Ed.), Advances in Experimental Social Psychology (Vol. 19, pp. 41-80). Academic Press, Inc.

Giancola, P. R., \& Zeichner, A. (1997). The biphasic effects of alcohol on human physical aggression. Journal of Abnormal Psychology, 106, 598-607.

Girandola, F. (2000a). Peur et persuasion : présentation des recherches et d'une nouvelle lecture. L'Ané'e psychologique, 100, 333-376.

Girandola, F. (2000b). Persuasion et résistance à la persuasion. Dans N. Roussiau (Ed.), Psychologie sociale (pp. 185-198). Editions In-Press.

Girandola, F. (2002). Persuasion et sante' publique. Dans C. Bonardi., F. Girandola., N. Roussiau., et N. Soubiale (Ed.), Psychologie sociale appliquée : environnement, sante', qualite' de vie (pp. 121-140).Editions In-Press.

Girandola, F. (2003). Psychologie de la persuasion et de l'engagement. Presses universitaires de Franche-Comte'.

Girandola, F. (2005). Action and Commitment theory. In. V. Alexandre., \& W. Gasparski (Eds.), French and other perspectives in praxeology (Vol. 12, pp. 117-130). Transaction Publishers, NJ.

Girandola, F., \& D. Atkinson. (2003). Preévention, détection et traitement de l'information en situation de peur. Revue canadienne des sciences du comportement, 35, 197-209.

Girandola, F., Joule, R.-V., \& Didelot, S. (2002). Effets de l'engagement sur l'implication persuasive : étude exploratoire. $4 \mathrm{e}$ Congrès de psychologie sociale en langue française, 1-4 septembre, Athènes, Grèce.

Girandola, F., \& Roussiau, N. (2003). L'engagement comme source de modifications à long terme. Les Cahiers Internationaux de Psychologie Sociale, 57, 83-101.

Goldberg, J. H., Halpern-Felsher, B. L., \& Millstein, S. G. (2002). Beyond invulnerability: The importance of benefits in adolescent's decision to drink alcohol. Health Psychology, 21, 477484. 
Howell, D. C. (1998). Méthodes statistiques en sciences humaines. DeBoeck Universite'.

Jaccard, J., \& Blanton, H. (2005). The origins and structure of behavior: Conceptualizing behaviour in attitude research. In D. Albarracin., B. T.

Johnson., \& M. P. Zanna (Eds.), The handbook of attitudes (pp. 125- 171). Mahwah: Erlbaum.

Johnson, B. T., Maio, G. R., \& Smith-McLallen, A. (2005). Communication and attitude change: Causes, processes, and effects. In D. Albarracin., B. T. Johnson., \& M. P. Zanna (Eds.), The handbook of attitudes (pp. 617-669). Mahwah: Erlbaum.

Joule, R. V. (1991a). Double forced compliance: A new paradigm in cognitive dissonance theory. Journal of Social Psychology, 131, 839-845.

Joule, R-V. (1991b). Practicing and arguing for abstinence from smoking: A test of the double forced compliance paradigm. European Journal of Social Psychology, 21, 119-129.

Joule, R-V., \& Beauvois, J-L. (1998). La soumission librement consentie. Dunod.

Joule, R-V., \& Beauvois, J-L. (2002). Petit traité de manipulation à l'usage des honnêtes gens. PUG.

Joule, R.V., Girandola, F., \& Bernard, F. (2007). How can people be induced to willingly change their behavior? The path from persuasive communication to committing communication. Social and Personality Psychology Compass, 1, 493-505.

Judd, C. M. (2000). Everyday data analysis in social psychology: Comparisons of linear models. In H. T., Reis., \& C. M. Judd (Eds.), Handbook of research methods in social and personality psychology (pp. 370-392). Cambridge University Press.

Kiesler, C. A. (1971). The psychology of commitment : Experiments linking behavior to belief. New York : Academic Press.

Kiesler, C. A., \& Sakumura, J. (1966). A test of a model of commitment. Journal of Personality and Social Psychology, 11, 321-327.

Lasswell, H. D. (1948). The structure and function of communication in society. In L. Bryson (Ed.), The communication of ideas: Religion and civilization series (pp. 37-51). New York: Harper and Row.

Leventhal, H. (1970). Findings and theory in the study of fear communications. Dans L. Berkowitz (Ed.), Advances in Experimental Social Psychology, 6, 199-186.

MacDonald, T. K., Zanna, M. P., \& Fong, G. T. (1998). Alcohol and intentions to engage in risky behaviors: Experimental evidence for a causal relationship. In J. G. Adair., D. Belanger., 
\& K. Dion (Eds.), Advances in psychological science: Vol. 1. Social, personal, and cultural aspects (pp. 407-428). East Sussex, UK : Psychology Press.

McNally, A. M., Palfai, T. P., \& Kahler, C. W. (2005). Motivational interventions for heavy drinking college students: Examining the role of discrepancy-related psychological processes. Psychology of Addictive Behaviors, 19, 79-87.

Michelik, F., \& Girandola, F. (2004). Résistance à la persuasion dans le paradigme de la communication engageante : effets sur la certitude de l'attitude. Actes du 5e Congrès International de Psychologie Sociale en Langue Française, Lausanne (Suisse), 1-4 septembre.

Miller, W. R., \& Rollnick, S. (2002). Motivational interviewing: Preparing people to change addictive behaviors. New York : Guilford Press.

Mongeau, P. (1998). Another look at fear arousing messages. In M. Allen, \& R. Preiss (Eds.), Persuasion: Advances through meta-analysis (pp. 53-68). Cresskill, NJ : Hampton Press.

Moscato, S., Black, D. R., Blue, C. L., Mattson, M., Galer-Unti, R. A., \& Coster, D. C. (2001). Evaluating a fear appeal message to reduce alcohol use among "Greeks". American Journal of Health Behavioral, 25, 481- 491.

Olson, J. M., \& Stone, J. (2005). The influence of behaviour on attitudes. In D. Albarracin., B. T. Johnson., \& M. P. Zanna (Eds.), The handbook of attitudes (pp. 223-271). Mahwah: Erlbaum.

Ouellette, J. A., \& Wood, W. (1998). Habit and intention in everyday life: The multiple processes by which past behaviour predicts future behaviour. Psychological Bulletin, 124, 54-74.

Perloff, R. M. (2001). Persuading people to have safer sex. Erlbaum.

Perloff, R. M. (2003). The dynamics of persuasion: Communication and attitudes in the 21st century. Erlbaum.

Petty, R. E., \& Cacioppo, J. T. (1986). Communication and persuasion: Central and peripheral routes to attitude change. Springer-Verlag.

Petty, R. E., \& Krosnick, J. A. (1995). Attitude strength: Antecedents and consequences. Erlbaum.

Petty, R. E., Priester, J. R., \& Briñol, P. (2002). Mass media attitude change: Implications of the Elaboration Likelihood Model of persuasion (pp. 155-198). In. J. Bryant., \& D. Zillmann (Eds.), Media effects: Advances in theory and research (pp. 155-198). Erlbaum.

Petty, R. E., \& Wegener, D. T. (1999). The elaboration likelihood model: Current status and controversies. In S. Chaiken., \& Y. Trope (Eds.), Dual-process theories in social psychology (pp. 41-72). Guilford Press. 
Rippetoe, P. A., \& Rogers, R. W. (1987). Effects of components of protection-motivation theory on adaptative and maladaptative coping with a health threat. Journal of Personality and Social Psychology, 52, 596-604.

Rogers, R. W. (1983). Cognitive and physiological processes in fear appeals and attitude change: A revised theory of protection motivation. Dans J. Cacioppo, \& R. Petty (Eds.), Social psychophysiology (pp. 153-176). New York : Guildford Press.

Rogers, R. W., \& Mewborn, C. R. (1976). Fear appeals and attitude change: Effects of a threat's noxiousness, probability of occurrence, and the efficacy of the coping responses. Journal of Personality and Social Psychology, 4, 54-61.

Ronis, D. L., Yates, J. F., \& Kirscht, J. P. (1989). Attitudes, decisions, and habits as determinants of repeated behaviour. In A. R. Pratkanis, S. J., Breckler, A. G. Greenwald (Eds.), Attitude structure and function (pp. 213-239). Hillsdale, NJ : Erlbaum.

Ruiter, R. A. C., Verplanken, B., De Cremer, D., \& Kok, G. (2004). Danger and fear control in response to fear appeals: The role of need for cognition. Basic and Applied Social Psychology, $26,13-24$.

Salancik, J. R., \& Kiesler, C. A. (1971). Behavioral commitment and retention of consistent and inconsistent attitude word-pairs: An experiment. In C. A. Kiesler (Ed.), The psychology of commitment: Experiments linking behavior to belief (pp. 111-121). Academic Press.

Sutton, S. (1994). The past predicts the future: Interpreting behaviour behaviour relationships in social psychological models of health behaviour. In D. R. Rutter., \& L. Quine (Eds.), Social psychology and health: European perspectives (pp. 71-88). Alderhost : Avebury.

Taubman Ben-Ari, O., Florian, V., \& Mikulincer, M. (2000). Does a threat appeal moderate reckless driving? A terror management theory perspective. Accident Analysis and Prevention, $32,1-10$.

Triandis, H. C. (1980). Values, attitudes, and interpersonal behaviour. In H. E. Howe., \& M. M. Page (Eds.), Nebraska Symposium on Motivation (pp. 195-259). Lincoln, NE : University of Nebraska Press.

Verplanken, B. (2006). Beyond frequency: Habit as mental construct. British Journal of Social Psychology, 45, 639-656.

Verplanken, B., Aarts, H.,\& van Knippenberg, A. (1997). Habit, information acquisition, and the process of making travel mode choices. European Journal of Social Psychology, 27, 539 560.

Verplanken, B., Myrbakk, V., \& Rudi, E. (2005). The measurement of habit. In T., Betsch., \& S. Haberstroh (Eds.), The routines of decision making (pp. 231-247). Mahwah, NJ : Erlbaum. 
Verplanken, B., \& Orbell, S. (2003). Reflections on past behavior: A self-report index of habit strength. Journal of Applied Social Psychology, 33, 1313-1330

Witte, K. (1992). The role of threat and efficacy in Aids prevention. International Journal of Community Health Education, 12, 225-249.

Witte, K. (1994). Fear control and danger control: A test of the extended parallel process model (EPPM). Communication Monographs, 61, 113- 134.

Witte, K. (1998). Fear as motivator, fear as inhibitor: Using the extended parallel process model to explain fear appeal successes and failures. In P. A. Andersen., \& L. K. Guerrero (Eds.), Handbook of communication and emotion : research, theory, applications, and contexts (pp. 423-450). Academic Press : San Diego.

Witte, K., \& Allen, M. (2000). A meta-analysis of fear appeals: Implications for effective public health campaigns. Health Education \& Behavior, 27, 591-615.

Witte, K., Cameron, K. A., McKeon, J. K., \& Berkowitz, J. M. (1996). Predicting risk behaviors: Development and validation of a diagnostic scale. Journal of Health Communication, 1, 317 341.

Witte, K., Meyer, G., \& Martell (2001). Effective health risk messages: A step-by-step guide. Sage Publication. 